\title{
SPINALE MUSKELATROPHIE - EIN UPDATE
}

Aliki Perdikari, Andrea Klein, David Jacquier

Übersetzer: Rudolf Schläpfer

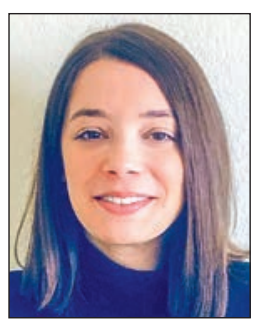

Aliki Perdikari

https://doi.org/ 10.35190/d2021.3.2

\section{Einführung}

Die spinale Muskelatrophie (SMA: Unter dieser Bezeichnung werden wir nur auf die «5q-assoziierte spinale Muskelatrophie» eingehen) ist eine progrediente neuromuskuläre Krankheit, welche die unteren Motoneurone befällt und zu einer zunehmenden Muskelschwäche führt. Lange Zeit eine der hauptsächlichsten genetischen Ursachen der Kindessterblichkeit, haben die wissenschaftlichen Fortschritte der letzten zwei Jahrzehnte die ersten spezifischen Behandlungen ermöglicht und zu einer Revolution in der Betreuung dieser Patienten geführt. Wir werden hier die Krankheit, die pathogenetischen Mechanismen, das klinische Bild und die derzeit verfügbaren therapeutischen Möglichkeiten besprechen.

\section{Genetik}

Mit einer Inzidenz von 1/6í000 bis 1/11í000 Lebendgeburten ist die SMA eine der häufigsten neuromuskulären Krankheiten ${ }^{1}$. Sie wird autosomal rezessiv vererbt. Der entscheidende pathogenetische Faktor ist der Mangel an «Survival Motor Neuron» (SMN) Protein, der wie es sein Name sagt, für das Überleben und die Entwicklung der Motoneurone unerlässlich ist, und während der Fetalperiode und den ersten Lebensmonaten eine entscheidende Rolle spielt. Das SMN-Protein wird beim Menschen ubiquitär exprimiert und ist an zahlreichen zellulären Vorgängen beteiligt ${ }^{2}$. Es sind nicht alle Gründe bekannt, weshalb die unteren Motoneurone bei dieser Krankheit im Vergleich zu anderen Zellarten besonders betroffen sind, und es ist wahrscheinlich, dass in Zukunft noch weitere zel- luläre Auswirkungen des Protein-SMN-Mangels ermittelt werden ${ }^{3)}$.

Das SMN-Protein hat seinen Ursprung in zwei paralogen, auf dem Chromosom $5 q$ liegenden Genen SMN1 und SMN2. Das aus 9 Exonen bestehende Gen SMN1 produziert den wesentlichen Anteil des vollständigen und funktionellen SMN-Proteins. Das Gen SMN2, das sich nur durch einige wenige Nukleotide von SMN1 unterscheidet und in einer variablen Anzahl Kopien (1 bis >4) vorhanden ist, kodiert zu $90 \%$ eine verkürzte und nicht funktionelle Form des SMN-Proteins (SMNCÊ̄7) und nur zu 10\% normales SMN-Protein ${ }^{2)}$ (Abbildung 1).

Die grosse Mehrheit (>95\%) der SMA-Patienten hat eine homozygote Deletion der Exone 7 und 8 (manchmal nur 7) des Gens SMN1, die übrigen haben eine punktuelle Mutation ${ }^{4}$. In der Mehrzahl der Fälle werden die Mutationen durch beide Eltern vererbt; die Trägerhäufigkeit erreicht je nach ethnischer Herkunft bis 1:501). Das Gen SMN2 stellt dann die einzige Quelle für das SMN-Protein dar, und es besteht ein umgekehrtes Verhältnis zwischen der Anzahl SMN2-Kopien und der Schwere der Krankheit ${ }^{5}$ ). In einer geringeren Anzahl Fälle können jedoch noch weitere modulierende Faktoren eine Rolle spielen ${ }^{3,6)}$.

\section{Klinisches Bild}

Die SMA besteht aus einem Kontinuum von Phänotypen, von der sehr schweren angeborenen (SMA 0) bis zur spät im Erwachsenenalter auftretenden Form
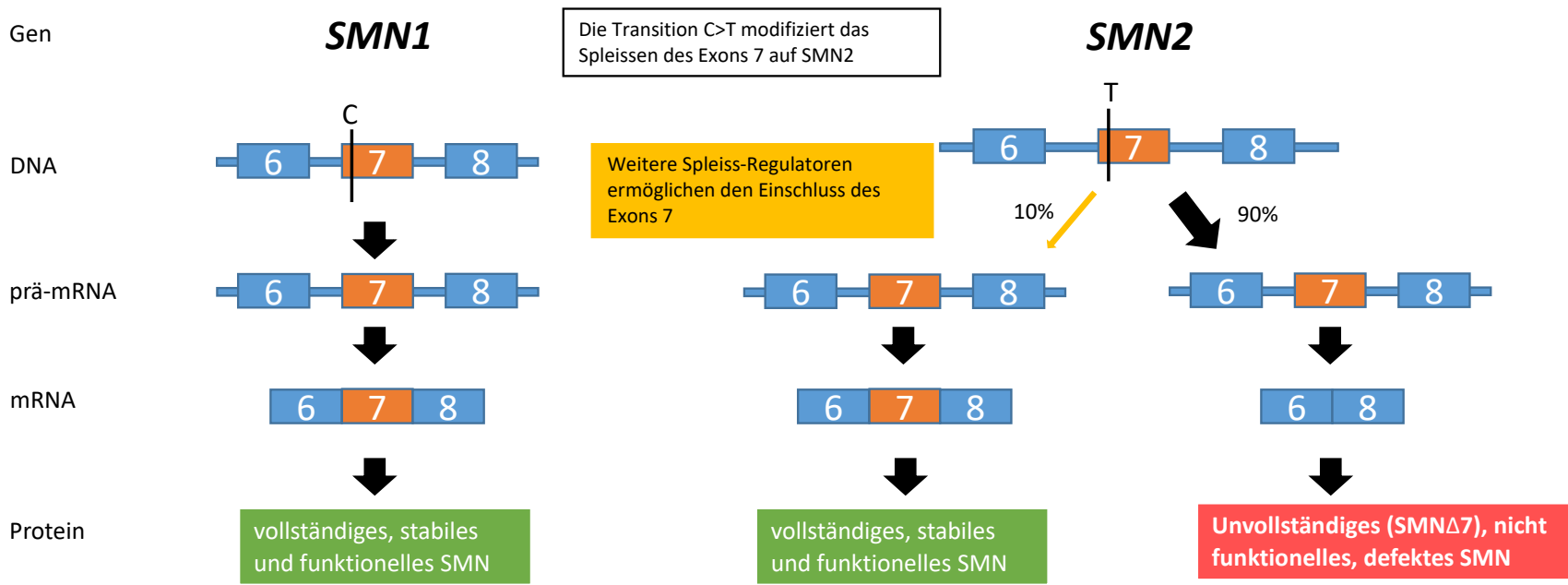

Abbildung 1. Schema zur Darstellung der unterschiedlichen Synthese des SMN-Proteins durch die Gene SMN1 und SMN2. mRNA: messenger RNA. Frei nach ${ }^{7)}{ }^{7}$ Messina S et Sframeli M. New Treatments in Spinal Muscular Atrophy: Positive Results and New Challenges. J Clin Med. 2020 Jul 13;9(7):2222 


\section{Fortbildung}

(SMA 4), wobei die Einteilung auf Grund des Alters beim Erscheinen der ersten Symptome und den besten erreichten motorischen Fähigkeiten erfolgt. Diese Klassifikation wurde durch das Einführen von Untergruppen (Buchstaben oder Dezimale) verfeinert, um die Verschiedenartigkeit der Erscheinungsbilder und Verläufe und schliesslich die Behandlungserfolge besser zu erfassen (Tabelle 1).

Alle SMA-Formen zeichnen sich durch das Auftreten einer progredienten Muskelschwäche aus, die vor allem die proximale (untere > obere) Extremitätensowie axiale Muskulatur betrifft. Die Sehnenreflexe verschwinden, es treten Faszikulationen der Zunge und Tremor der Hände auf. Der Befall der Atem-, insbesondere Interkostalmuskulatur führt zu paradoxer Atmung, wenig wirksamem Husten, progredienter restriktiver Ventilationsstörung und Atelektasen. Auch das Kauen und Schlucken sind betroffen. Es treten gastrointestinale Störungen und sehr häufig orthopädische Probleme, insbesondere Skoliose, auf. Kognitive und sensorische Störungen treten nicht auf, und nur sehr selten ein kardialer Befall. Forschung und günstigere Überlebenschancen besonders von Patienten mit schweren SMA-Formen weisen jedoch vermehrt darauf hin, dass die SMA eine multisystemische Krankheit ist ${ }^{3}$.

\section{Diagnose}

Die Möglichkeit einer SMA muss bei jedem Säugling oder Kind in Betracht gezogen werden, die eine symmetrische, proximal und/oder an den unteren Extremitäten betonte, insbesondere progrediente Muskelschwäche ohne allgemeine Entwicklungsverzögerung aufweisen. Faszikulationen der Zunge und Tremor der Hände sind ebenfalls ein wichtiger Hinweis. Die CK kann normal oder erhöht sein (selten $>1000 E /$ ). Muskelbiopsie und ENMG sind nicht erforderlich, obwohl letztere Untersuchung sich bei spät und schleichend auftretenden Formen als nützlich erweisen kann. Es ist dann wichtig, das Kind schnellstmöglich an eine(n) Neuropädiater(in) oder direkt an ein Zentrum für neuromuskuläre Erkrankungen im Kindes- und Jugendalter zu überweisen.

Die entscheidende Etappe besteht in der genetischen Untersuchung einer Blutprobe zur Bestimmung der homozygoten Exon 7- und 8-Deletion im SMN1Gen, oft durch MLPA- oder qPCR-Methode. Die Sequenzierung des Gens zur Identifizierung von punktuellen Mutationen ist selten notwendig ${ }^{9}$. Die Bestimmung der AnzahI SMN2-Kopien wird heutzutage häufig parallel durchgeführt.

\section{Betreuung}

Gemäss den internationalen Empfehlungen ${ }^{9,10}$ wird die multidisziplinäre Betreuung durch ein Zentrum für neuromuskuläre Erkrankungen im Kindes- und Jugendalter, in Zusammenarbeit mit Neuro- und Praxispädiatrie in die Wege geleitet. Es sind zahlreiche medizinische Spezialfächer (Pneumologie, Orthopädie, Gastroenterologie, usw.) involviert eine therapeutische Betreuung (Physio- und Ergotherapie, Psychologie, usw.) sowie auch weitere Akteure wie Schule, Sozialdienst oder Patientenvereinigungen, um nur einige zu nennen.

Seit 2008 sammelt das Schweizer Register für neuromuskuläre Erkrankungen Swiss-Reg-NMD (www. swiss-reg-nmd.ch) auf freiwilliger Basis Daten zu Patienten (Erwachsene und Kinder) mit SMA, um die epidemiologische Entwicklung verfolgen zu können, zu Forschungs- und therapeutischen Zwecken und insbesondere, um den Verlauf unter spezifischen Therapien, auf die wir unten eingehen werden, beurteilen zu können.

\begin{tabular}{|c|c|c|c|c|}
\hline $\begin{array}{l}\text { SMA-Typ } \\
\text { Anteil Fälle }\end{array}$ & $\begin{array}{l}\text { Alter bei } \\
\text { Erscheinen der } \\
\text { ersten Symptome }\end{array}$ & $\begin{array}{l}\text { Maximale } \\
\text { motorische } \\
\text { Entwicklung }\end{array}$ & $\begin{array}{l}\text { Motorische und } \\
\text { andere Aspekte }\end{array}$ & $\begin{array}{l}\text { Prognose bei } \\
\text { «natürlicher Entwicklung» }\end{array}$ \\
\hline $\begin{array}{l}\text { SMA } 0 \\
<1 \%\end{array}$ & Fetal & Fehlend & $\begin{array}{l}\text { Schwerste Hypotonie, } \\
\text { Arthrogrypose, neonatale } \\
\text { Ateminsuffizienz. }\end{array}$ & $\begin{array}{l}\text { Exitus im Verlaufe } \\
\text { der ersten Wochen }\end{array}$ \\
\hline $\begin{array}{l}\text { SMA } 1 \\
50 \%\end{array}$ & $\begin{array}{l}<2 \text { Wochen }(1 \mathrm{a}) \\
<3 \text { Monate }(1 \mathrm{~b}) \\
<6 \text { Monate }(1 \mathrm{c})\end{array}$ & $\begin{array}{l}\text { Fehlend } \\
\text { (1c Kopfkontrolle } \\
\text { möglich) }\end{array}$ & $\begin{array}{l}\text { Schwere Hypotonie, } \\
\text { Ateminsuffizienz, } \\
\text { Schluckstörung }\end{array}$ & $\begin{array}{l}\text { Exitus oder Dauerbeatmung } \\
(>16 \mathrm{Std} / \mathrm{d}) \text { vor dem Alter } \\
\text { von } 2 \text { Jahren }\end{array}$ \\
\hline $\begin{array}{l}\text { SMA } 2 \\
30 \%\end{array}$ & 6-18 Monate & $\begin{array}{l}\text { Sitzen (selten } \\
\text { stehen möglich) }\end{array}$ & $\begin{array}{l}\text { Proximale Schwäche, } \\
\text { Skoliose, Atembeschwerden }\end{array}$ & $\begin{array}{l}\text { Lebenserwartung bis 3.-5. } \\
\text { Jahrzehnt, je nach Befall/ } \\
\text { Beatmungsstrategie }\end{array}$ \\
\hline $\begin{array}{l}\text { SMA } 3 \\
20 \%\end{array}$ & $\begin{array}{l}<3 \text { Jahre }(3 a) \\
3-12 \text { Jahre }(3 b) \\
\text { 12-18 Jahre }(3 c)\end{array}$ & Gehen & $\begin{array}{l}\text { Verlust der Gehfähigkeit } \\
\text { (Typ 3a/b), Skoliose und } \\
\text { Atembeschwerden möglich }\end{array}$ & $\begin{array}{l}\text { Lebenserwartung } \\
\text { beinahe normal } \\
\text { (insbesondere Typ 3b) }\end{array}$ \\
\hline $\begin{array}{l}\text { SMA } 4 \\
<1 \%\end{array}$ & Erwachsen & Normal & «Leichte» Muskelschwäche & Lebenserwartung normal \\
\hline
\end{tabular}

Frei nach ${ }^{8)}$ Farrar MA, Park SB, Vucic S, Carey KA, Turner BJ et al. Emerging therapies and challenges in spinal muscular atrophy. Ann Neurol 2017 Mar;81(3):355-68 


\section{Fortbildung}

Gezielte medikamentöse Behandlung

Im September 2017 wurde Nusinersen (Spinraza ${ }^{\circ}$ ), das erste spezifische Medikament gegen SMA, durch Swissmedic bewilligt. Es handelt sich um ein Antisense-Oligonukleotid, das den Einschluss des Exons 7 beim Spleissen des SMN2-Gens verbessert und so die Produktion an komplettem und funktionellem SMA-Protein fördert; je mehr SMN2-Kopien, desto höher wird die Produktion an SMN-Protein sein ${ }^{11)}$. Dieses Medikament wird intrathekal verabreicht in altersund gewichtsunabhängiger Dosis. Das Behandlungsschema sieht 4 Aufsättigungsdosen an den Tagen 0-14-28-63 vor, anschliessend eine Erhaltungsdosis alle vier Monate, je nach Wirksamkeit; letztere bestimmt auch die Vergütung durch die IV aufgrund strikter Kriterien.

Zwei weitere Medikamente wurden kürzlich durch Swissmedic bewilligt. Risdiplam (Evrysdi ${ }^{\circ}$ ) ist wie Nusinersen ein Spleissmodulator des SNM2-Gens mit dem Ziel, die Produktion an komplettem SMN-Protein zu erhöhen ${ }^{12)}$. Es handelt sich um eine trinkbare, täglich einzunehmende Lösung mit systemischer Wirkung, die ausschliesslich auf die Motoneurone ausgerichtet ist. Risdiplam wurde in der Schweiz im Mai 2021 für Kinder mit SMA ab dem Alter von 2 Monaten bewilligt, wobei die Anwendungs- und Vergütungsbedingungen in den kommenden Monaten genauer angegeben werden.

Das zweite Medikament ist Onasemnogene abeparvovec-xioi (Zolgensma'), dessen Wirkungsmechanismus von SMN2 unabhängig ist. Es handelt sich um eine Gentherapie, die einen nicht-replizierenden adenoassoziierten viralen Vektor (AAV9) benutzt, um ein Nukleosom in die Zellkerne einzuführen, das nicht in die DNA integriert wird und ein kontinuierliches Äquivalent des SMN1-Gens zu exprimieren vermag ${ }^{13}$. Das Medikament wird intravenös verabreicht, wirkt systemisch und gemäss den aktuellen Kenntnissen nach einer einmaligen Dosis über Jahre. Es wurde über mögliche schwere $\mathrm{Ne}$ benwirkungen berichtet, die eine weitere Überprüfung erfordern ${ }^{15,16)}$. Zolgensma ${ }^{\circledR}$ wurde in der Schweiz im Juni 2021 für Kinder mit SMA ab dem Alter von 2 Monaten bewilligt. Man wird auch da die nächsten Monate abwarten müssen, um die genauen Anwendungs- und Vergütungsbedingungen zu erfahren.

Es ist aus Platzgründen nicht möglich, hier auf die verschiedenen Wirksamkeitsstudien einzugehen. Kurz zusammengefasst ermöglichen die drei Medikamente eine Verbesserung der motorischen Funktionen und das Erreichen wichtiger Meilensteine (Sitzen, Stehen, Gehen), sowie die Verlängerung von Lebensdauer und Überleben ohne anhaltenden Ventilationsbedarf; dies bei Säuglingen mit SMA Typ 1, was in eindeutigem Gegensatz zum natürlichen Verlauf steht. Nusinersen und Risdiplam sind auch bei Patienten mit anderen SMA-Formen und verschiedenen Alters (auch Erwachsenen) wirksam. Wesentlich für das Ausmass des Behandlungserfolges ist die Dauer der Krankheit. Die sehr hohen Kosten der drei Medikamente bedingen strikte Betreuung und Nachkontrollen, bezüglich Anwendung der Medikamente sowie therapeutische Wirkung. Wir laden Sie ein, den Artikel von Dangouloff und Servais zu lesen, der die Studien dieser drei Medikamente gut zusammenfasst ${ }^{17}$.

\section{Neugeborenen-Screening?}

Die möglichst frühzeitige Wiederherstellung eines genügend hohen Protein SMN-Spiegels erlaubt es, die Motoneuronen zu bewahren, und ist der Eingriff mit dem eindeutig besten Behandlungseffekt und der bestmöglichen motorischen Entwicklung. Eine präsymptomatische Behandlung ist nicht nur möglich, sondern auch vorzuziehen, wie es die Studien für Nusinersen (NURTURE, klinische Studie NCT02386553, publiziert ${ }^{18)}$ ), Risdiplam (Rainbowfish, klinische Studie NCT03779334) und Onasemnogene abeparvovec-xioi (SPR1NT, Studie NCT03505099) zeigen. Diese Tatsachen sprechen für eine Implementierung des neonatalen SMA-Screenings. Ein solches Screening wird in verschiedenen Ländern weltweit entwickelt, ein Dutzend Länder haben es bereits implementiert (z.B. Deutschland anfangs 2021). Überlegungen dazu sind in der Schweiz im Gange. Das Thema wird bestimmt in Paediatrica zu gegebener Zeit ausführlicher behandelt werden.

\section{Fazit}

Wir erleben grosse Veränderungen in der Betreuung der SMA, mit wesentlichen Elementen die bewahrt werden (z.B. multidisziplinäre Betreuung), Neuerungen, die implementiert werden müssen (z.B. Medikamente), und Fragen die Antworten erfordern (z.B. Langzeitverlauf). Die Zukunft wird deshalb sehr reich an Überlegungen und Herausforderungen sein.

Für das Literaturverzeichnis verweisen wir auf unsere Online Version des Artikels.

\section{Autoren}

Dr med Aliki Perdikari, Unité de neurologie et neuroréhabilitation pédiatrique, Centre hospitalier universitaire vaudois (CHUV), Lausanne

Dr med Andrea Klein, Département de neurologie pédiatrie, Hôpital universitaire pédiatrique de Bâle (UKBB), Bâle et Hôpital universitaire pédiatrique de Berne, Hôpital de l'île, Berne Dr med David Jacquier, Unité de neurologie et neuroréhabilitation pédiatrique, Centre hospitalier universitaire vaudois (CHUV), Lausanne

Die Autoren haben keine finanziellen oder persönlichen Verbindungen im Zusammenhang mit diesem Beitrag deklariert. 